\title{
Simulation Study of Individual Thermal Comfort with the Integrated Personalized Fan and VAV System
}

\author{
Siliang Lu ${ }^{1}$, Erica Cochran Hameen ${ }^{1}$ \\ ${ }^{1}$ School of Architecture, Carnegie Mellon University, Pittsburgh, USA
}

\begin{abstract}
Variable Air Volume (VAV) system has been widely used in commercial buildings, especially open-plan office buildings for years. Compared to private offices, openplan offices are faced with problems like too much interruptions and unsatisfactory shared indoor temperature and humidity. Since HVAC system aims to create comfortable thermal environment, it is of great importance to develop new paradigm of HVAC system framework so that everyone could work under their preferred thermal environment. Therefore, this paper aims to investigate thermal comfort improvements by operating an integrated personalized fan and VAV system in an open-plan office. A field study was conducted to develop adaptive thermal comfort models with Random forest (RF) classification, and CFD simulation with Ansys Icepak 18.2 was conducted to analyze the airflow patterns and thermal stratifications. As a result, the recall scores of the two thermal comfort models are $73.3 \%$ and $76.5 \%$, respectively. Moreover, the simulation results have shown that the comfort level with the integrated system in the occupied zone is higher than that with the baseline as the temperature set-point increases.
\end{abstract}

\section{Introduction}

As defined by ANSI/ASHRAE 55 (2013) and ISO7730 (2011), thermal comfort is the condition of mind that expresses satisfaction with the thermal environment and is assessed by subjective evaluation. Therefore, thermal comfort is the combined result of physical environment and psychological activities. In the course of thermal comfort theory, static thermal comfort and adaptive thermal comfort have become two main categories. In terms of static thermal comfort, predicted mean vote (PMV) developed by Fanger (1970) is the most widely accepted thermal model where seven variables are accepted to be the indicators of thermal comfort, which are indoor air temperature, indoor relative humidity, indoor air velocity, mean radiant temperature, clothing insulation, metabolic rate and external work (normally 0 ). However, PMV was derived from a strictly controlled climate chamber where the thermal environment was static and different from the real office environment. Moreover, since PMV predicts the average vote of a large group of people based on the seven-point thermal sensation scale, instead of individual thermal comfort, it only describes the overall thermal sensation of multiple occupants in a shared thermal environment.

In order to overcome the disadvantages of static thermal comfort, adaptive thermal comfort aims to provide insights in highlighting individual thermal comfort, which increases opportunities for personal and responsive control, thermal comfort enhancement, energy consumption reduction and climatically responsive and environmentally responsible building design (De Dear et al., 1998). One of the milestone projects of adaptive thermal comfort is the ASHRAE RP-884 (De Dear et al., 1998), which collected a total of 22000 sets of data from the real office environments across the world. This project has been widely used to investigate various adaptive thermal models, which have been integrated into personalized HVAC controls.

In addition, in order to investigate advanced statistical methods to establish adaptive thermal comfort models, a recent research utilized the wearable device, which was a pair of eyeglass frame with infrared (IR) arrays to infer thermal comfort. The results demonstrated $82.8 \%$ of prediction accuracy for detecting uncomfortable conditions was obtained with hidden markov model (HMM) (Ghahramani et al., 2018). Moreover, Huang et al. (2015) also used wearable devices like wearable fitness trackers to measure physical movements, sweat level and skin temperature so as to infer individual thermal comfort with machine learning algorithms including random forest (RF) and support vector machine (SVM). In addition, Dai et al. (2017) implemented SVM to predict thermal demands using skin temperature collected from various locations with wearable sensors. As a result, SVM classifiers with linear kernel were preferred to Gaussian kernel, which achieved over $90 \%$ accuracy. Based on the literature review, classification models have performed so well as regression models.

Among various HVAC systems optimizing energy performances and thermal comfort, task ambient conditioning (TAC) system is one of the most promising systems. TAC is defined as any space conditioning system that allows thermal conditions in small, localized zones to be individually controlled by building occupants, while still automatically maintaining acceptable environmental conditions in the ambient space of the building (Bauman et al., 1996). Due to the advantages of TAC that not only 
takes individual thermal preferences into account but also maintains the overall acceptable thermal environment, TAC has become one of the popular lighting systems in open-plan office buildings. However, since HVAC system is more complicated to operate than lighting system due to many factors such as complex airflow patterns and thermal lag, TAC for HVAC system hasn't been explored deeply, such as integrating the adaptive thermal models discussed above into TAC system so as to enhance operational performances. Zhang et al. (2010) developed a task-ambient system that heated only the feet and hands, and cooled only the hands and face, to provide comfort in a wide range of ambient environment. The simulated annual heating and cooling energy savings with such TAC system was as much as $40 \%$. Moreover, personal comfort system (PCS) is an innovation to integrate low-energy PCS to create micro-zones into centralized HVAC operations in open plan office environments (Anderson et al., 2015; Bauman et al, 2017). The project has invented the low-energy heated and cooled chairs with wireless internet connectivity and tested the performances in different real office environments in California. The PCS adjusts the local thermal environment based on occupant behavioural models of changing heating/cooling set-points of the chair. Meanwhile, the whole framework gets further optimized with communication between chairs and the centralized HVAC system. In a case study, the test energy performances were optimized with the mode of widening HVAC temperature set-point dead band in conjunction with PCSs. In addition, among various TAC devices, fans are usually selected as personalized cooling systems under the framework of TAC due to their costeffectiveness and higher efficiency than existing airconditioning system such as VAV. The fans there offer a straight-forward, economic, independently operable and most importantly, effective technique to increase movement of air so as to ultimately improve thermal comfort in a room (Sekhar et al., 1995). This is generally done by removing/circulating the warm stratified air from/in the room. Moreover, when operated with AC system, the downwash propelled by foil (rotating) drives the supply air to blend with the existing air, mitigating the uncomfortable thermal stratification. Moreover, since widening the set-point dead band reduces sensible loads, the energy consumption can be reduced. Therefore, minimizing temperature difference between the indoor and outdoor air temperature or maximizing heating and cooling dead band reduces energy consumption while it may compromise occupant thermal comfort. Fortunately, since the personalized cooling system like fan provides additional cooling with little draft due to low air velocity. Hence, the integration of the centralized cooling system and the personalized system increases comfortable cooling set-point, thus achieving energy savings and maintaining thermal comfort.

Besides empirical studies on TAC, a few studies have been conducted to analyse thermal comfort with simulation tools such as CFD. Sekhar et al. (2018) proposed an integrated personalized ventilation and local fan-induced active chilled beam (PV-ACB) air conditioning system and was analysed based on thermal stratification with CFD. The results have shown that the integrated system is able to achieve occupant thermal comfort. Moreover, (Makhoul et al., 2013) conducted a computational fluid dynamic (CFD) simulation to study the enhancement of ceiling mounted personalized ventilation (PV) when assisted by desktop fans to reduce thermal plume. Thermal comfort models were developed based on skin temperature, core temperature, as well as sensible and latent heat flux calculated with multisegmented bioheat model. The bioheat model were cosimulated with CFD model by exchanging data related to thermal environment, including mean radiant temperature, air temperature and air velocity. The simulation results have shown the desk-mounted fans were able to reduce the convection plumes around the occupant and achieved energy saving by up to $13 \%$ when compared with conventional mixing ventilation systems. However, both of them used static PMV-PPD model, which may not be appropriate to evaluate thermal comfort in real office environment.

In order to evaluate thermal comfort with more realistic thermal models and study the proposed integrated personalized cooling fan and VAV system in a more detailed way by including airflow pattern and thermal stratification analysis, this paper aims to not only simulate the TAC system with CFD but also apply the two adaptive thermal models based on an empirical study conducted in a regular open-plan office in Shanghai during cooling season into CFD simulation.

\section{Methods}

\section{Development of adaptive thermal models}

Figure 1 shows the diagram of the integrative study with data collection in the test bed and CFD simulation. As shown in the figure, the individual thermal comfort models with RF were developed based on a six-day empirical study in an open-plan office in Shanghai with subtropical climate. Meanwhile, a CFD simulation with the same building configurations, including floorplan, envelopes, air-conditioning system as the test bed used in the empirical study, were conducted to analyse effects of increase temperature set-point dead band on individual thermal comfort with the individual thermal comfort models developed with the empirical study.

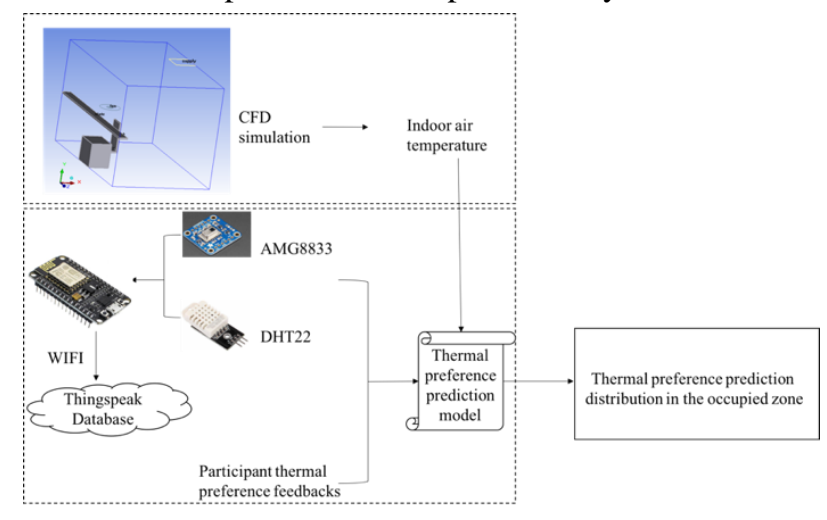


Figure 1: The diagram of the method for the proposed local fan-induced air-conditioning

The thermal models were developed based on a six-day empirical study with two subjects (one male and one female) between $8 / 21 / 2018$ and $8 / 27 / 2018$ in Shanghai. The male subject had 171 instances while the female subject had 151 instances. The objective of the experiment is to predict thermal comfort of the two participants with the proposed TAC framework under different thermal environment conditions with accuracy as high as possible so that the two thermal models can be used for thermal comfort analysis with the data generated from CFD simulations. Based on the design of nonintrusive sensing system and the literature review of principle factors to thermal comfort, the measured variables are shown in the following table. The interval of data collection is 5 minutes, which corresponds with the survey responses. In all, the whole components in the personalized cooling system includes the sensing system and an 8' desktop mini-fan (the supply direction can be adjusted and the air velocity in the occupied zone can be controlled under $0.25 \mathrm{~m} / \mathrm{s}$ ). Even if the sensing system can also be used for the automatic personalized heating control, this study is only focused on cooling season. Figure 2 shows the current prototype of the personalized system.



Figure 2: Proposed sensing system

Table 1: Measured variables and the measurement tools

\begin{tabular}{|l|l|}
\hline Variable & Measurement tool \\
\hline Local air temperature & DHT22 \\
\hline Local relative humidity & DHT22 \\
\hline Cheek skin temperature & AMG8833 \\
\hline
\end{tabular}

Meanwhile, in order to create different thermal environment conditions, an overhead air conditioner was controlled in transient conditions where the air temperature increases from $24{ }^{\circ} \mathrm{C}$ to $30{ }^{\circ} \mathrm{C}$. To avoid discomfort due to high air velocity and asymmetric radiation, the participants were asked to adjust locations of their working areas if they could feel draft and if they could feel the sunlit before measurement.

Meanwhile, the participants were asked to provide feedback regarding their current thermal sensation with 7point sensation scale. Even if neutral sensation doesn't necessary mean thermal comfort, it is a precondition of comfort (Auliciems et al. 1997). Therefore, the statement that occupant feels thermally comfortable when they feel the thermal environment is neutral was in this study, and 7-point thermal sensation scale as classification labels was used, as shown in Table 2.

Table 2: ASHRAE thermal sensation scale

\begin{tabular}{|c|c|}
\hline Value & Meaning \\
\hline-3 & very hot \\
\hline-2 & hot \\
\hline-1 & warm \\
\hline 0 & neutral \\
\hline 1 & cool \\
\hline 2 & cold \\
\hline 3 & very cold \\
\hline
\end{tabular}

In addition, Figure 3 shows a participant status in the empirical study. The fan was located at the upper level in the occupied zone to avoid draft due to high air velocity. Before each session, the participants' clothes were reported and heart rates were measured so as to ensure the participants have similar regular status in the beginning.



Figure 3: Empirical study setup with a participant

The development of thermal models follows the typical machine learning pipeline, as shown in Figure 4, including data pre-processing, model selection, model training, model testing and real-time prediction.

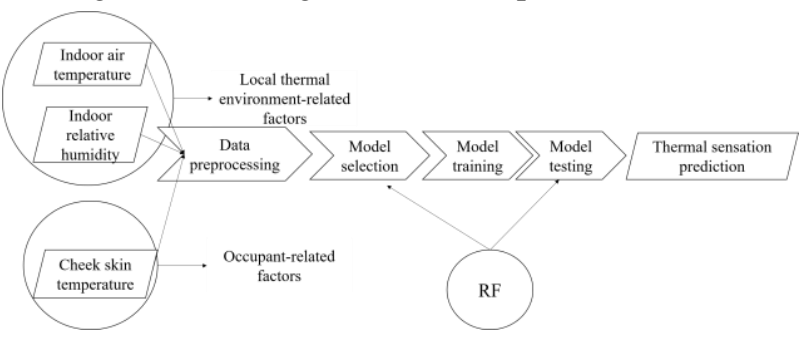

Figure 4: Thermal comfort model pipeline

The whole experimental data was split into two parts where $90 \%$ of data is the training set and $10 \%$ is the 
testing data. In addition, before training the best model, the hyper-parameters used in the best models are selected with 5-fold cross-validation (Kohavi, 1995).

In terms of classification algorithms, rather than neural network, Random forest (RF) was implemented for classification since RF can perform well with such relatively small-scale dataset. RF is an ensemble learning method that averages the accuracy of a number of decision trees constructed with bagging algorithm at training time. However, random forest adds additional randomness to the model that it looks for the most important feature among a random subset of features, random subset of the whole dataset instead of all features, and the whole dataset while splitting the node. Compared to building deep decision trees to train high-dimensional data, owing to randomness, RF builds several smaller trees, thus suffering less from overfitting (Liaw et al., 2002). As a result, it generally can develop better model than decision trees. In this study, the number of trees and the tree depth were also selected with cross-validation.

The prediction results of RF were compared with PMV model and all the thermal models were evaluated based on recall, precision and F1 scores (Goutte et al., 2005), which were calculated with the confusion matrix, as shown in Table 3. Recall score measures the ability of a model to find all correctly classified instances within a dataset, which is the ratio between true positives and all actual positives. Precision score describes how precise the model is out of the predicted results, which is the ratio between true positives and all predicted positives. Lastly, F1 score is used for seeking balance between recall and precision, which is harmonic mean of recall and precision. In terms of multi-label classification, the weighted average of recall, precision and F1 score are calculated, which might result in an F1 score that is not between recall and precision.

Table 3: Confusion matrix

\begin{tabular}{|l|l|l|}
\hline & $\begin{array}{l}\text { Predicted } \\
\text { negative }\end{array}$ & Predicted true \\
\hline $\begin{array}{l}\text { Actual } \\
\text { negative }\end{array}$ & True negative & True positive \\
\hline Actual positive & False negative & False positive \\
\hline
\end{tabular}

\section{Development of CFD simulation}

In order to analyse effects of the personalized cooling system on the overall indoor thermal environments and individual thermal comfort, a computational fluid dynamic (CFD) simulation was conducted to study thermal stratifications and adaptive thermal comfort with the proposed fan-induced air-conditioning system in an open-plan office. The simulation was conducted with Ansys Icepak 18.2, which provides powerful electronic cooling solutions utilizing ANSYS Fluent solver for thermal and fluid flow analyses. Figure 5 shows the 3D model where a fan is located on the plane above the occupant while the overall thermal environment was controlled with the supply air and the workstation is built in the middle of the room left side.

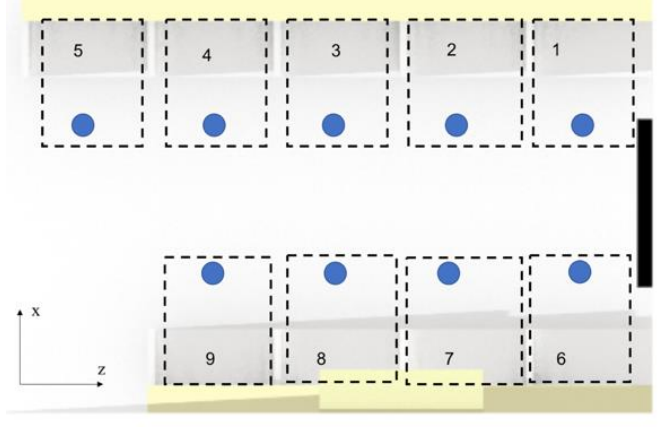

Figure 5: Spot measurement mapping

The model has the same setup as the empirical study with Icepak 18.2 where a fan is located on the plane above the occupant while the overall thermal environment was controlled with the supply, as shown in Figure 6.

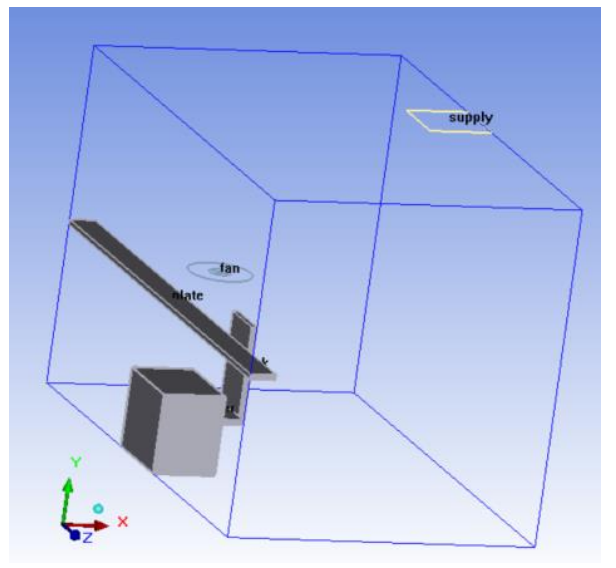

Figure 6: 3D simulation model

Besides the setup of the integrated TAC system, the occupant was simulated as a solid heat source with a constant power of $100 \mathrm{~W}$ and supply terminal was simulated as a free opening with high supply velocity being $5 \mathrm{~m} / \mathrm{s}$. The occupied zone is defined as the region within an occupied space between 75 and $1800 \mathrm{~mm}$ above the floor and more than $600 \mathrm{~mm}$ from the walls or fixed air-conditioning equipment (Burroughs \& Hansen, 2013). The relative humidity of the supply air was set as a constant value of $50 \%$. In addition, the outdoor temperature was set to be $30^{\circ} \mathrm{C}$ and one side of the room was simulated as a window with higher heat transfer coefficient than other stationary walls. Lastly, $k-w$ SST turbulence model and Semi-Implicit Method for Pressure Linked Equations (SIMPLE) algorithm was used to solve Navier-Stokes equations. Meanwhile, the hexahedral unstructured mesh was generated. With the individual thermal comfort models developed with the empirical study and CFD simulation, the thermal comfort distribution with or without fans in the occupied zone can be simulated and compared. However, since the skin temperature cannot be simulated in the model and the relative humidity is set to be constant, the adaptive thermal comfort models used in the post-processing analysis of CFD simulation were developed with the air temperature only. 


\section{Results}

\section{Results of adaptive thermal models}

Based on cross-validation, Table 5 shows the selected hyper-parameter values for RF. Moreover, PMV was used as a baseline. Several assumptions were set when calculating PMV. Firstly, since the mean radiant temperature (MRT) and indoor air velocity were not measured, MRT was approximated with indoor air temperature when calculating PMV and the indoor air velocity was calculated as $0.1 \mathrm{~m} / \mathrm{s}$. Moreover, since the subjects were seated during the whole study, the metabolic rate was calculated as 1 met. Lastly, clothing insulation was set as 0.5 and there was no external work.

Table 5: Hyper-parameters used in RF

\begin{tabular}{|c|c|}
\hline Number of trees & 100 \\
\hline Tree depths & 3 \\
\hline
\end{tabular}

Meanwhile, Table 6 and Table 7 show the performance benchmark among the thermal models of the two subjects trained by RF and PMV. As a result, for the female subject, recall, precision, and $\mathrm{F} 1$ scores of $\mathrm{RF}$ on test data are $73.3 \%, 63.1 \%$, and $67.8 \%$, respectively. Compared to the baseline of PMV, RF have better performances. For the male subject, recall, precision and F1 scores of RF on test data are $76.5 \%, 76.3 \%$ and $74.4 \%$, respectively, which also outperform the baseline.

Table 6: Benchmark of the female thermal model

\begin{tabular}{|l|l|l|l|}
\hline & Recall & Precision & F1 \\
\hline RF & $73.3 \%$ & $63.1 \%$ & $67.8 \%$ \\
\hline PMV & $33.3 \%$ & $57.4 \%$ & $33.4 \%$ \\
\hline
\end{tabular}

Table 7: Benchmark of the male thermal model

\begin{tabular}{|l|l|l|l|}
\hline & Recall & Precision & F1 \\
\hline RF & $76.5 \%$ & $76.3 \%$ & $74.4 \%$ \\
\hline PMV & $23.5 \%$ & $54.6 \%$ & $21 \%$ \\
\hline
\end{tabular}

\section{Results of CFD simulation}

The following table shows the validation results of the simulation with the empirical study under the temperature set-point of $24^{\circ} \mathrm{C}$. The standard deviation of the spot measurement and the simulation result difference is 0.04 , which indicates the simulated indoor thermal environment matches the empirical study and the thermal environment can be used to predict individual thermal comfort.

Table 8: Validation of the air velocities $[\mathrm{m} / \mathrm{s}$ ]

\begin{tabular}{|l|l|l|l|l|l|l|l|l|l|}
\hline & $\mathbf{1}$ & $\mathbf{2}$ & $\mathbf{3}$ & $\mathbf{4}$ & $\mathbf{5}$ & $\mathbf{6}$ & $\mathbf{7}$ & $\mathbf{8}$ & $\mathbf{9}$ \\
\hline Actual & 0.3 & 0.35 & 0.35 & 0.1 & 0.1 & 0.15 & 0.1 & 0.06 & 0.02 \\
\hline Simulated & 0.35 & 0.4 & 0.44 & 0.08 & 0.08 & 0.08 & 0.08 & 0.04 & 0.02 \\
\hline
\end{tabular}

Meanwhile, Figure 7-8 show the temperature contours with or without operation of the fan at the cross-section where the fan is located with the temperature set-point being $24^{\circ} \mathrm{C}$, respectively. Meanwhile, Figure 9-10 show the temperature contours with or without operation of the fan at the same plan with the temperature set-point being $30^{\circ} \mathrm{C}$. As shown in the figure, when the air temperature set-point is $24^{\circ} \mathrm{C}$, the supply air is induced in the occupied zone so as to overcome thermal plumes with the help of the fan. However, when the temperature set-point increases further, there is no difference in the air temperature distribution with or without fan.

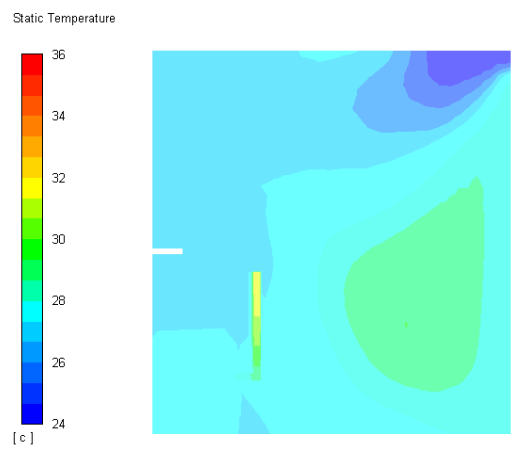

Figure 7: The operation with fan when set-point $=24^{\circ} \mathrm{C}$

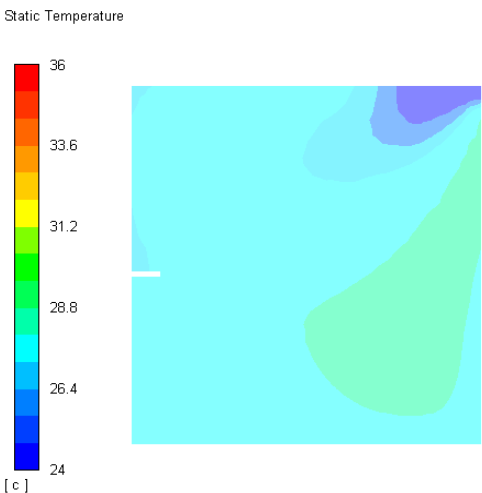

Figure 8: The operation without fan when setpoint $=24^{\circ} \mathrm{C}$

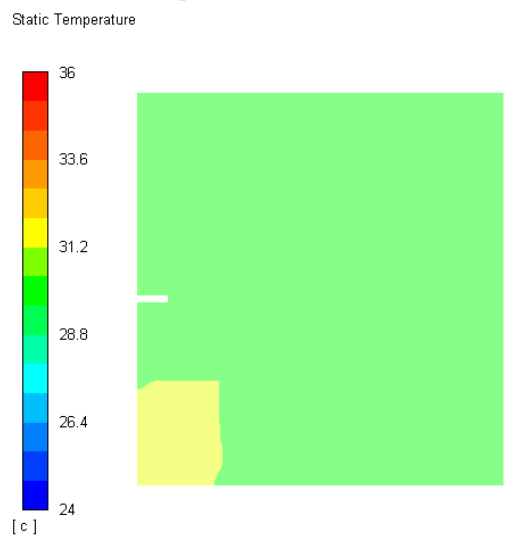

Figure 9: The operation with fan when set-point $=30^{\circ} \mathrm{C}$ 
Static Temperature
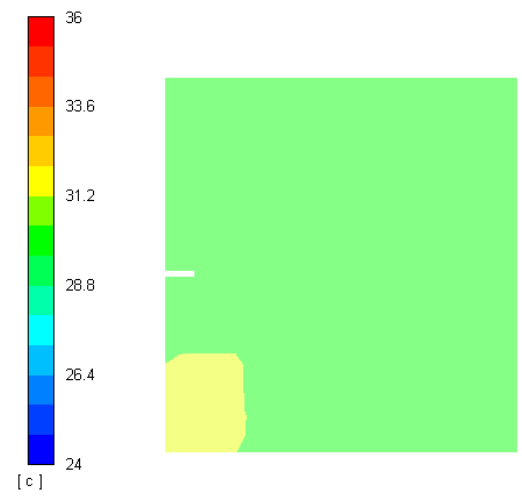

Figure 10: The operation without fan when setpoint $=30^{\circ} \mathrm{C}$

In addition, Table 9 shows the proportion of area at the cross-section where the fan is located in the occupied zone (height is smaller than $0.8 \mathrm{~m}$ since the occupant is seated) where the thermal sensation prediction of each subject is neutral with or without the fan when the temperature setpoint increases from $24^{\circ} \mathrm{C}$ to $30^{\circ} \mathrm{C}$. As shown in the table, the proportion of neutral sensation of both subjects in the occupied zone with the proposed TAC system is no smaller than that without fans as temperature set-point increases until $28^{\circ} \mathrm{C}$. This indicates the proposed system with fan operation indeed can increase set-point dead band while maintain individual thermal comfort. However, when the temperature set-point increases to $30{ }^{\circ} \mathrm{C}$, the advantage of fan operation no longer exists since there is no cool supply air in the occupied zone.

Table 9: Benchmark of the proportion of the neutral sensation

\begin{tabular}{|c|c|c|c|c|c|c|c|c|}
\hline & \multicolumn{2}{|c|}{$24^{\circ} \mathrm{C}$} & \multicolumn{2}{c|}{$26^{\circ} \mathrm{C}$} & \multicolumn{2}{c|}{$28^{\circ} \mathrm{C}$} & \multicolumn{2}{c|}{$30^{\circ} \mathrm{C}$} \\
\hline & $\mathrm{m}$ & $\mathrm{f}$ & $\mathrm{m}$ & $\mathrm{f}$ & $\mathrm{m}$ & $\mathrm{f}$ & $\mathrm{m}$ & $\mathrm{f}$ \\
\hline with & 92 & 92 & 83 & 83 & 75 & $75 \%$ & 0 & 0 \\
& $\%$ & $\%$ & $\%$ & $\%$ & $\%$ & $\%$ & $\%$ & $\%$ \\
\hline witho & 92 & 92 & 83 & 83 & 45 & 45 & 0 & 0 \\
ut & $\%$ & $\%$ & $\%$ & $\%$ & $\%$ & $\%$ & $\%$ & $\%$ \\
\hline
\end{tabular}

Moreover, Figure 11 shows the air temperature change along the fan axis from 0 to $1.5 \mathrm{~m}$ height in the occupied zone, which further validates the advantage of using personalized fan. As shown in the figure, when the height is larger than $0.75 \mathrm{~m}$ and the temperature setpoint is no more than $28^{\circ} \mathrm{C}$, under the same temperature setpoint, the air temperature without fan operation is higher than that with fan operation at the same height level. Moreover, the temperature difference with or without fan increases as the temperature setpoint increases. As a result, there is little difference with or without fan operation when the temperature setpoint increases to $30^{\circ} \mathrm{C}$. In addition, the temperature differences between the ankle and neck under different temperature set-points are all less than $3{ }^{\circ} \mathrm{C}$ to ensure thermal comfort while the temperature gradient increases suddenly at the height of $0.8 \mathrm{~m}$ due to the heat generation of occupants.

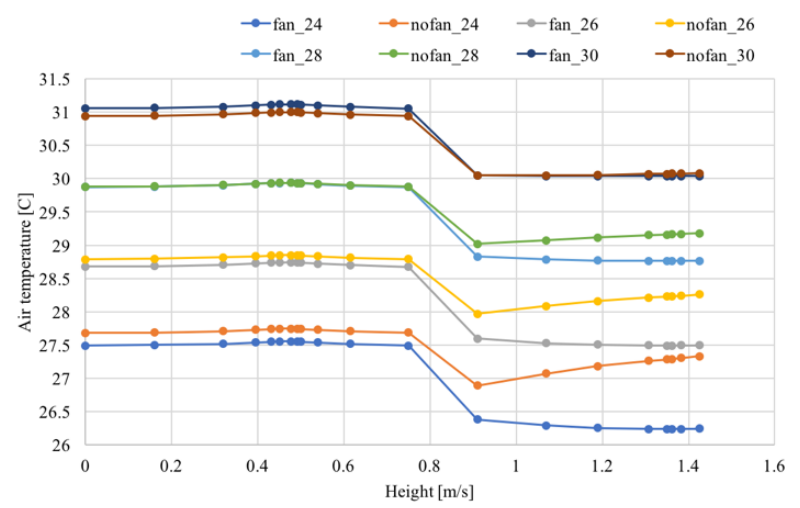

Figure 11: The air temperature change along the fan axis from 0 to $1.5 \mathrm{~m}$ height in the occupied zone

\section{Conclusions}

This paper aims to investigate thermal comfort improvements by operating an integrated personalized fan and VAV system in an open-plan office. A field study was conducted to develop adaptive thermal comfort models with Random forest (RF) classification, and CFD simulation with Ansys Icepak 18.2 was conducted to analyze the airflow patterns and thermal stratifications with the proposed system. As a result, the recall scores of the two thermal comfort models are $73.3 \%$ and $76.5 \%$, respectively. Moreover, the simulation results have shown that the comfort level with the proposed integrated system in the occupied zone is higher than that with the baseline as the temperature set-point increases. Therefore, the proposed integration of the personalized cooling system and the centralized VAV system turns out to achieve energy savings while maintain individual thermal comfort.

\section{Acknowledgement}

The first author of the paper is supported by China Scholarship Council.

\section{References}

Andersen, M. P., Fierro, G., Kumar, S., Chen, M., Truong, L., Kim, J., Arens E.A., Zhang H., Raftery P., \& Culler, D. E. (2015). Well-connected microzones for increased building efficiency and occupant comfort. In Proceedings of the 2nd ACM International Conference on Embedded Systems for EnergyEfficient Built Environments.

ASHRAE, A. S. (2013). Standard 55-2013. Thermal environmental conditions for human occupancy, 12.

Auliciems, A., \& Szokolay, S. V. (1997). Thermal comfort. PLEA.

Bauman, F., Raftery, P., Kim, J., Kaam, S., Schiavon, S., Zhang, H., Arens, E., Brown, K., Peffer, T., Blumstein, C. \& Culler, D. (2017). Changing the Rules: Innovative Low-Energy Occupant-Responsive HVAC Controls and Systems. 
Bauman, F. S., \& Arens, E. A. (1996). Task/ambient conditioning systems: engineering and application guidelines.

Burronughs, H.E. \& Hansen Shirley J. (2013). Managing indoor air quality.

Dai, C., Zhang, H., Arens, E., \& Lian, Z. (2017). Machine learning approaches to predict thermal demands using skin temperatures: Steady-state conditions. Building and Environment, 114, 1-10.

De Dear, R., \& Brager, G. S. (1998). Developing an adaptive model of thermal comfort and preference.

Fanger, P. O. (1970). Thermal comfort. Analysis and applications in environmental engineering. Thermal comfort. Analysis and applications in environmental engineering.

Ghahramani, A., Castro, G., Karvigh, S. A., \& BecerikGerber, B. (2018). Towards unsupervised learning of thermal comfort using infrared thermography. Applied Energy, 211, 41-49.

Goutte, C., \& Gaussier, E. (2005). A probabilistic interpretation of precision, recall and F-score, with implication for evaluation. In European Conference on Information Retrieva. Springer, Berlin, Heidelberg.

Huang, C. C. J., Yang, R., \& Newman, M. W. (2015). The potential and challenges of inferring thermal comfort at home using commodity sensors. In Proceedings of the 2015 ACM International Joint Conference on Pervasive and Ubiquitous Computing.
Kohavi, R. (1995). A study of cross-validation and bootstrap for accuracy estimation and model selection. In IJCAI.

Liaw, A., \& Wiener, M. (2002). Classification and regression by randomForest. R news, 2(3), 18-22.

Makhoul, A., K. Ghali, and N. Ghaddar (2013). "Desk fans for the control of the convection flow around occupants using ceiling mounted personalized ventilation." Building and Environment, 59 (2013): 336-348.

Roelofsen, P. (2011). Evaluation of draught in surgical operating theatres: proposed revision to (NEN)-ENISO-7730. Journal of Facilities Management, 9(1), 6470.

Sekhar, Chandra, and Long Zheng (2018). "Study of an integrated personalized ventilation and local faninduced active chilled beam air conditioning system in hot and humid climate." In Building Simulation, vol. 11, no. 4, pp. 787-801. Tsinghua University Press, 2018.

Zhang, H., Arens, E., Kim, D., Buchberger, E., Bauman, F., \& Huizenga, C. (2010). Comfort, perceived air quality, and work performance in a low-power taskambient conditioning system. Building and Environment, 45(1), 29-39. 